\title{
Structure and Reactivity of Surface Oxides on Pt(110) during Catalytic CO Oxidation
}

\author{
M. D. Ackermann, ${ }^{1,2}$ T. M. Pedersen, ${ }^{3}$ B. L. M. Hendriksen, ${ }^{2}$ O. Robach, ${ }^{4}$ S. C. Bobaru, ${ }^{2}$ I. Popa, ${ }^{1}$ C. Quiros, ${ }^{5}$ H. Kim, ${ }^{1}$ \\ B. Hammer, ${ }^{3}$ S. Ferrer, ${ }^{6}$ and J. W. M. Frenken ${ }^{2}$ \\ ${ }^{1}$ ESRF, 6, rue Jules Horowitz, F-38043 Grenoble cedex, France \\ ${ }^{2}$ Kamerlingh Onnes Laboratory, Leiden University, P.O. Box 9504, 2300 RA Leiden, The Netherlands \\ ${ }^{3}$ Interdisciplinary Nanoscience Center (iNANO) and Department of Physics and Astronomy, University of Aarhus, \\ DK-8000 Aarhus C, Denmark \\ ${ }^{4}$ CEA-Grenoble DRFMC/SI3M/PCM17, rue des Martyrs, 38054 Grenoble cedex 9, France \\ ${ }^{5}$ Departamento de Física, Faculdad de Ciencias, Universidad de Oviedo, Avda. Calvo Sotelo, s/n, 33007 Oviedo, Spain \\ ${ }^{6}$ CELLS - ALBA, Edifici Ciències Nord. Mòdul C-3 centra, Campus Universitari de Bellaterra, Universita Autònoma de Barcelona,
} 08193 Bellaterra, Spain

(Received 1 July 2005; published 16 December 2005)

\begin{abstract}
We present the first structure determination by surface $\mathrm{x}$-ray diffraction during the restructuring of a model catalyst under reaction conditions, i.e., at high pressure and high temperature, and correlate the restructuring with a change in catalytic activity. We have analyzed the $\operatorname{Pt}(110)$ surface during $\mathrm{CO}$ oxidation at pressures up to 0.5 bar and temperatures up to $625 \mathrm{~K}$. Depending on the $\mathrm{O}_{2} / \mathrm{CO}$ pressure ratio, we find three well-defined structures: namely, (i) the bulk-terminated $\operatorname{Pt}(110)$ surface, (ii) a thin, commensurate oxide, and (iii) a thin, incommensurate oxide. The commensurate oxide only appears under reaction conditions, i.e., when both $\mathrm{O}_{2}$ and $\mathrm{CO}$ are present and at sufficiently high temperatures. Density functional theory calculations indicate that the commensurate oxide is stabilized by carbonate ions $\left(\mathrm{CO}_{3}^{2-}\right)$. Both oxides have a substantially higher catalytic activity than the bulk-terminated Pt surface.
\end{abstract}

DOI: 10.1103/PhysRevLett.95.255505

PACS numbers: 61.10.Eq, 82.65.+r

$\mathrm{CO}$ oxidation on Pt single crystal surfaces forms a favorite model system for heterogeneous catalysis. Most of our present-day understanding of this system is based on a large number of experiments that have been carried out under laboratory rather than industrial conditions, such as ultrahigh vacuum (UHV) and low temperatures. Presently, a growing number of experimental techniques is being adapted to bridge the so-called pressure gap and obtain atomic-scale insight in the structure, composition, and other properties of model catalyst surfaces under (semi) realistic reaction conditions. Examples are scanning tunneling microscopy [1-3], surface x-ray diffraction [4-7], transmission electron microscopy [8], and photoelectron spectroscopy [9].

Previous high-pressure STM observations during CO oxidation on $\operatorname{Pt}(110)$ have demonstrated that at a sufficiently high $\mathrm{O}_{2} / \mathrm{CO}$ pressure ratio this surface undergoes a structural phase transition, which has a dramatic effect on the reaction mechanism and strongly enhances the reaction rate [2]. The STM movies have been interpreted in terms of the formation of a thin platinum oxide film on the $\mathrm{Pt}(110)$ surface, on which the $\mathrm{CO}$ molecules oxidize through a Mars-Van Krevelen mechanism, and react with $\mathrm{O}$ atoms from the oxide. There is a growing body of evidence from experiments [2,10,11] as well as theory [12] strongly suggesting that similar scenarios are active for $\mathrm{CO}$ oxidation on other metal surfaces.

In this Letter we present the first observation by highpressure surface $\mathrm{x}$-ray diffraction of a correlation between the reactivity and the structure of a catalyst surface. The experiment performed during $\mathrm{CO}$ oxidation on $\mathrm{Pt}(110)$ shows that the most active structures are, in fact, two thin oxide film configurations, one of which is stable only when the surface is in contact with both reactant gasses, $\mathrm{CO}$ and $\mathrm{O}_{2}$, at elevated temperatures.

The experiments were performed at the ID03 beam line of the European Synchrotron Radiation Facility (ESRF) in a combined UHV-high-pressure surface $\mathrm{x}$-ray diffraction (SXRD) chamber $\left(10^{-10} \mathrm{mbar}-2\right.$ bar), which had a volume of $\sim 2$ liter and was equipped with a $360^{\circ}$ beryllium window for entrance and exit of the $\mathrm{x}$ rays [13]. The sample could be heated up to $1300 \mathrm{~K}$ in vacuum. Connected to the chamber were a gas manifold with four high-purity gasses (N47 grade for CO, N55 for all other) and a quadrupole mass spectrometer (QMS) for online gas analysis. The chamber was mounted on a $z$-axis diffractometer with the crystal surface in a horizontal plane.

A focused beam of monochromatic, $17 \mathrm{keV}$ x-ray photons was impinging on the surface at an angle of $1^{\circ}(\sim 4 \times$ $10^{12}$ photons/s). The fluorescence radiation in the scattered beam was filtered with a crystal analyzer. We describe the Pt crystal lattice with two perpendicular unit vectors $\mathbf{A}_{1}$ and $\mathbf{A}_{2}$ in the surface plane along [110] and [001], respectively, and the third one, $\mathbf{A}_{3}$, along the [110] surface normal. $\left|\mathbf{A}_{\mathbf{1}}\right|=a_{0}=2.774 \AA$ is the Pt nearestneighbor distance; $\left|\mathbf{A}_{\mathbf{2}}\right|=\sqrt{ } 2 a_{0}$ and $\left|\mathbf{A}_{\mathbf{3}}\right|=a_{0}$. In reciprocal space $\mathbf{H}$ and $\mathbf{K}$ are the unit vectors in the surface plane (parallel to $\mathbf{A}_{1}$ and $\mathbf{A}_{2}$, respectively) and $\mathbf{L}$ is along the surface normal. Well-ordered, clean $\operatorname{Pt}(110)$ surfaces exhibiting the characteristic $(1 \times 2)$ missing-row recon- 
struction [14] were obtained after several ion bombardment $\left(1 \mathrm{keV} \mathrm{Ar}^{+}\right)$and annealing $(1200 \mathrm{~K})$ cycles. The full width at half maximum (FWHM) of a rocking scan around the $\left(\begin{array}{lll}0 & 0.5 & 0.2\end{array}\right)$ reflection was typically $0.24^{\circ}$, which corresponds to ordered domain sizes of $\sim 1200 \AA$.

Our procedure to form a well-ordered oxide film was to first lift the $(1 \times 2)$ missing-row reconstruction by exposing the surface to 100 mbar of $\mathrm{CO}$ at $600 \mathrm{~K}$ [15]. Within minutes, the surface reordered to a $(1 \times 1)$ bulk-terminated surface with terraces of $\sim 3000 \AA$. The surface was then cooled to room temperature under $\mathrm{CO}$ to preserve the $(1 \times 1)$ structure. Subsequently, the CO pressure was reduced to 10 mbar and 500 mbar of $\mathrm{O}_{2}$ was admitted to the chamber, after which the sample was heated to $650 \mathrm{~K}$. This procedure led to new diffraction peaks at noninteger $\mathbf{H}$ and $\mathbf{K}$ values (see Fig. 1) forming a regular, slightly distorted hexagonal pattern, exhibiting angles of $57.4^{\circ}$ and $61.3^{\circ}$. As indicated in Fig. 1, the hexagons were aligned with respect to the substrate crystallographic directions. The intensities of the new reflections were similar to those of the surface reflections from the clean substrate, which immediately indicated that they arose from one or more

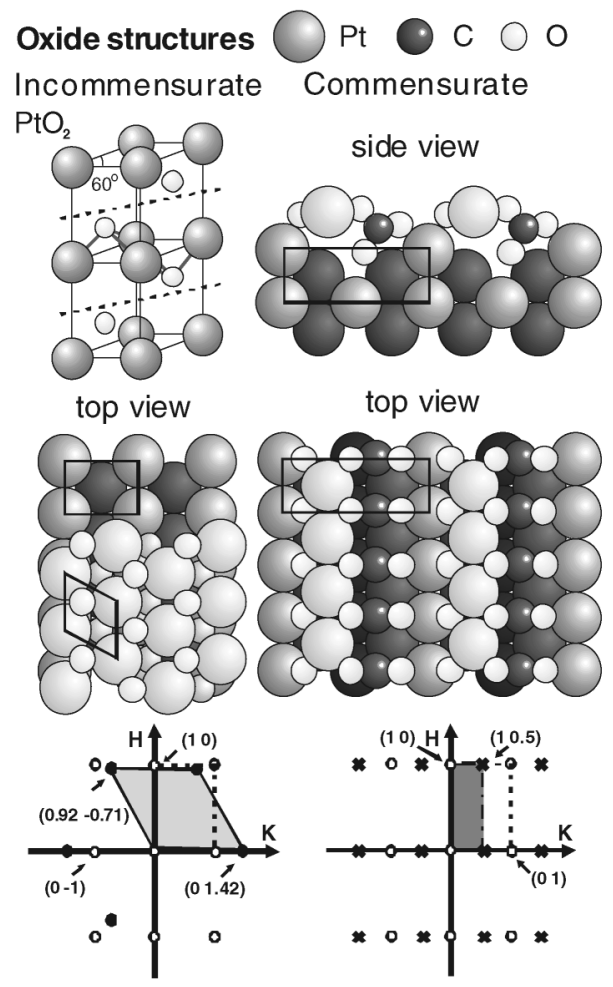

FIG. 1. Bottom left: in-plane reciprocal space map of the $\operatorname{Pt}(110)$ substrate (open circles), with the incommensurate, quasihexagonal oxide overlayer (solid circles). One diffraction peak could not be measured due to instrumental limitations. Higher orders have been measured as well (not shown). Bottom right: the $(1 \times 2)$, commensurate oxide overlayer (crosses). All coordinates are expressed in reciprocal lattice units of $\operatorname{Pt}(110)$. The ball models show the real-space structures of the two oxides. layers of Pt atoms, rather than from an overlayer of merely $\mathrm{O}$ atoms or $\mathrm{CO}$ molecules. The unit mesh of the nearly hexagonal Pt layer was very close to that of $\alpha-\mathrm{PtO}_{2}$ (see Fig. 1), suggesting that the overlayer was a distorted $\mathrm{PtO}_{2}$ layer, azimuthally aligned with the substrate, and oriented with its $c$ axis parallel to the surface normal [16]. Relative to the bulk lattice of $\alpha-\mathrm{PtO}_{2}$ our surface oxide exhibited compressive strain of $3.4 \%$ along the [110] direction and tensile strain of $2.4 \%$ along the [001] direction. We have also oxidized the $(1 \times 2)$-reconstructed surface without preexposure to $\mathrm{CO}$. The resulting oxide layers exhibited the same structure, but with very poor order [3], both in and out of plane. Hence, all experiments discussed here were performed with oxide layers grown on the $(1 \times 1)$ bulkterminated surface.

A crystallographic data set consisting of 22 in-plane reflections (up to 4 th order, 10 nonequivalent) and 3 crystal truncation rods ( 2 nonequivalent, Fig. 2) was collected to determine the oxide structure. The oscillations along $\mathbf{L}$ in the data in Fig. 2 show that several Pt layers were involved in the surface oxide. For a quantitative fit to the intensities [17], we started from a distorted $\alpha-\mathrm{PtO}_{2}$ film and had as free parameters (i) the thickness of the oxide, (ii) the lattice constant along $\mathbf{A}_{3}$, and (iii) the distance between the oxide film and the substrate. As the scattering of the $\mathrm{x}$ rays is largely dominated by the Pt atoms, the stoichiometry and the relative positions of the oxygen atoms were set to those for the bulk oxide. The continuous curves in Fig. 2 correspond to $2.6 \pm 0.1$ oxide monolayers (ML), i.e., a 40:60 mixture of 2- and 3-ML oxide films, an oxide monolayer being defined as an O-Pt-O triple layer. The fitted Pt-Pt distance is $2.8 \AA$ along $\mathbf{A}_{3}$, which is $36 \%$ shorter than the reported distance in bulk $\mathrm{PtO}_{2}$. The fit to the $(00 \mathrm{~L}) \operatorname{rod}$ (Fig. 2) confirms this rather high density of the oxide layer compared to reported values for bulk $\mathrm{PtO}_{2}$. This strong contraction might be due to the very small thickness of the oxide film. The distance between the outermost Pt layer of the substrate and the innermost Pt layer of the oxide was found to be $3.3 \pm 0.1 \AA$, which is very close to the Pt-PtO distance calculated by Helveg et al. for a fully oxidized Pt atom row on the missing-row reconstructed $\mathrm{Pt}(110)$ surface [18].

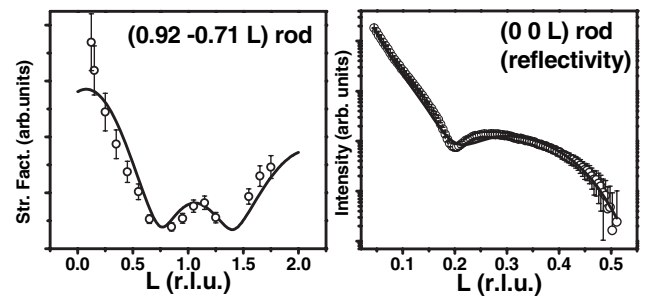

FIG. 2. Structure factor at $(\mathbf{H}, \mathbf{K})=(0.92,-0.71)$ as a function of $\mathbf{L}$ (left), and reflection intensity curve [(00L)-rod] (right), measured at $625 \mathrm{~K}$ and 0.5 bar of $\mathrm{O}_{2}$ on $\mathrm{Pt}(110)$. $\mathrm{L}$ is expressed in reciprocal lattice units of the substrate. The solid curves are a fit for the incommensurate, $2.6 \mathrm{ML}, \alpha-\mathrm{PtO}_{2}$ oxide film. 
Simultaneously with the diffraction measurements we have recorded the $\mathrm{CO}$ oxidation reactivity both on the bulkterminated metal surface and on oxidized surfaces. These experiments were performed in the so-called batch mode, in which the chamber was first filled with 500 mbar of $\mathrm{O}_{2}$, with the Pt sample at a temperature in the range 425$625 \mathrm{~K}$. SXRD measurements showed that under these conditions the surface was covered with the $\alpha-\mathrm{PtO}_{2}$ structure discussed above. Pulses of pure $\mathrm{CO}$ were then introduced into the chamber [e.g., at point (a) in Fig. 3]. The CO started reacting to $\mathrm{CO}_{2}$ as soon as it entered the chamber. From the decrease in the $\mathrm{CO}$ and $\mathrm{O}_{2}$ partial pressures, $\mathrm{P}_{\mathrm{CO}}$ and $\mathrm{P}_{\mathrm{O}_{2}}$, and the increase in the $\mathrm{CO}_{2}$ partial pressure, $\mathrm{P}_{\mathrm{CO}_{2}}$, the typical turnover number can be estimated to be approximately $3 \times 10^{3}$ molecules/site/s at $625 \mathrm{~K}$ in a mixture of 80 mbar $\mathrm{CO}$ and $500 \mathrm{mbar}_{2}$. The comparison of the turnover numbers from experiments at different temperatures suggests that the maximum reaction rate was limited by the diffusion of $\mathrm{CO}$ through the $\mathrm{O}_{2}$ dominated gas mixture to the surface and not by the reactivity of the catalyst surface. Several other groups have found similarly high turnover numbers in comparable conditions $[19,20]$.

In the top panel of Fig. 3 we show the diffracted intensity at $\left(\begin{array}{lll}0 & 1.42 & 0.5\end{array}\right)$ from the oxide layer. The introduction of CO caused a sudden $\sim 25 \%$ drop in the intensity followed by a slow recuperation. During the recovery, the shape of the $(01.42 \mathrm{~L})$ scan and the in-plane FWHM of the reflection did not change significantly, indicating that the surface remained covered by an equally thick oxide layer throughout the reaction. The intensity drop is ascribed to a roughening of the oxide layer caused by the reaction, which leads to a growing density of pits and protrusions, an effect that has already been seen by STM under similar conditions [2]. Because of the incommensurability of the oxide layer, the in-plane domain size is very small $(\sim 7-20 \mathrm{~nm}$ depending on growth conditions) and it does not reduce further during the roughening. The fifth $\mathrm{CO}$ pulse, at point (b) in Fig. 3,

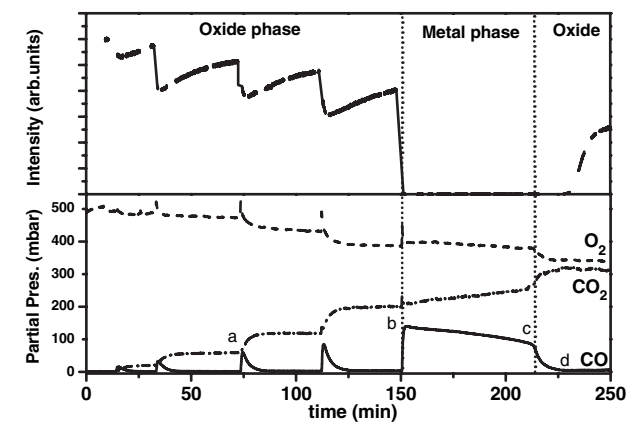

FIG. 3. Simultaneously measured x-ray diffraction intensity at $(0,1.42,0.5)$ from the quasihexagonal oxide (top panel) and partial pressures of $\mathrm{CO}, \mathrm{O}_{2}$, and $\mathrm{CO}_{2}$ (bottom panel). Separate $\mathrm{CO}$ pulses were admitted to the reactor, which was initially filled with 500 mbar of $\mathrm{O}_{2}$ at a temperature of $625 \mathrm{~K}$. The sharp peaks in $\mathrm{P}_{\mathrm{O}_{2}}$ are an artefact due to the sudden increase of total pressure at each $\mathrm{CO}$ pulse. was so large that the oxide layer was reduced completely: the diffracted intensity from the oxide layer dropped to zero and the surface reverted to the bulk-terminated $(1 \times 1)$ periodicity, found before in pure CO. A simultaneous decrease was observed in the reactivity, by a factor of roughly 25 , as calculated from the decay rate of $\mathrm{P}_{\mathrm{CO}}$ after (b) with respect to the decay rates after the preceding pulses. Also the reaction order was changed, since after (b) $\mathrm{P}_{\mathrm{CO}}$ varied roughly linearly in time, i.e., with a rate that was independent of the amount of remaining $\mathrm{CO}$, whereas it decayed exponentially, i.e., with a rate proportional to $\mathrm{P}_{\mathrm{CO}}$, after the four earlier pulses when the surface was still covered by the oxide.

At point (c) in Fig. 3, at a partial pressure ratio $\mathrm{P}_{\mathrm{CO}} / \mathrm{P}_{\mathrm{O}_{2}}$ of approximately 0.2 , the $\mathrm{CO}_{2}$ formation rate sped up and the variation in all partial pressures switched to exponential again, indicating that the catalyst had reverted to its "highreactivity" phase. Interestingly, the diffraction intensity associated with the incommensurate oxide overlayer came back only at point (d), 20 minutes after (c).

As Fig. 4 shows, immediately when the reaction sped up, new diffraction peaks appeared at integer $\mathbf{H}$ values and half-integer $\mathbf{K}$ values, corresponding to a commensurate $(1 \times 2)$ structure (Fig. 1), rather than the incommensurate, quasihexagonal oxide structure, developing later at point (d). The intensities of the new diffraction peaks were high, indicating that also these were due to a structure involving Pt atoms. Figure 5 shows $\mathbf{L}$ scans of two of the new halforder reflections. They clearly differ from the calculated values expected for the $(1 \times 2)$-reconstructed, clean $\operatorname{Pt}(110)$ surface [14], indicating that the structure differed strongly from the known missing-row reconstruction. The solid curves in Fig. 5 show the fit of the $\mathbf{L}$ scans, based on a simple structural model in which every second closepacked [110] row of surface Pt atoms was strongly dis-

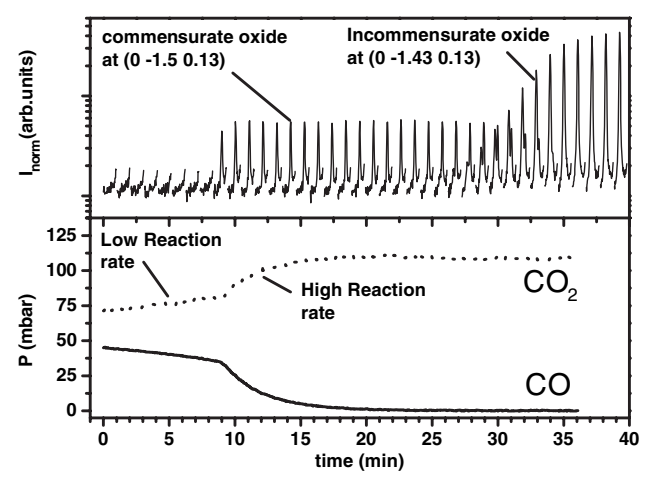

FIG. 4. Diffraction scans in a narrow range from $\mathbf{K}=-1.30$ to -1.65 and at $(\mathbf{H}=0, \mathbf{L}=0.13)$ (top panel), and partial pressures of $\mathrm{CO}$ and $\mathrm{CO}_{2}$, simultaneously measured at $\mathrm{P}_{\mathrm{O}_{2}}=$ 500 mbar and $T=625 \mathrm{~K}$. Together with the increase in the reaction rate, at $t=9 \mathrm{~min}$, a half-order diffraction peak appeared at $\mathbf{K}=-1.50$. When nearly all $\mathrm{CO}$ had been consumed, at $t \approx 30 \mathrm{~min}$, this diffraction peak decreased to zero while the peak of $\alpha-\mathrm{PtO}_{2}$, at $\mathbf{K}=-1.43$, appeared. 

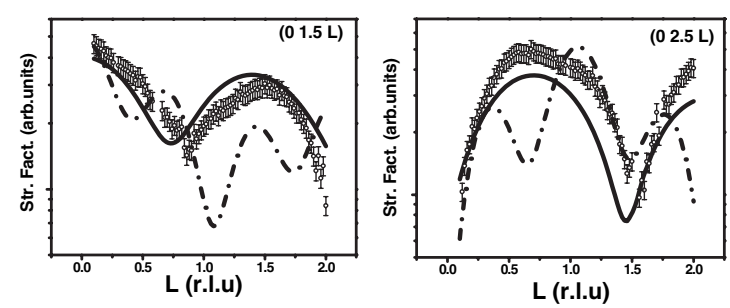

FIG. 5. The $(01.5 \mathrm{~L})$ and $(02.5 \mathrm{~L})$ rods of the $(1 \times 2)$ commensurate oxide layer, formed at $625 \mathrm{~K}$ and 0.5 bar of $\mathrm{O}_{2}$ and approximately 35 mbar of $\mathrm{CO}$ on $\mathrm{Pt}(110)$. The solid curves are fits for the carbonate-stabilized commensurate oxide film, as discussed in the text. The dash-dotted lines are calculated curves for the $1 \times 2$ missing-row reconstruction [14].

placed. In addition to this large displacement, small relaxations of all other atoms in the two topmost layers of $\mathrm{Pt}$ were considered. Relaxations beyond this second layer did not add any accuracy to the fit, and were hence neglected, as were the $\mathrm{C}$ and $\mathrm{O}$ atom positions. In the best-fit structure every second Pt surface atom row was displaced by $(0.5-0.380 .67)$, expressed in unit vectors of the $(1 \times 1)$ unit cell, while the relaxations of the other atoms were modest (Fig. 1). With the low-symmetry position of the shifted $\mathrm{Pt}$ atom rows, two equivalent mirror versions can be formed, and the best fit was obtained for nearly equal proportions of these.

Although the two surface oxide structures resolved here are different from the structures observed in Ref. [3] after exposure of $\mathrm{Pt}(110)$ to atomic oxygen, some of the structural elements might be similar. In particular, local configurations in Figs. 1(b)-(d) of Ref. [3] resemble the geometry of the commensurate surface oxide, discussed above.

The observation that the commensurate oxide structure was only stable under reaction conditions, when both $\mathrm{CO}$ and $\mathrm{O}_{2}$ were present, strongly suggests that the $(1 \times 2)$ oxide was stabilized by the presence of $\mathrm{CO}$ or a CO-related species, adsorbed on the oxide surface. In order to further substantiate this notion we have performed density functional theory calculations, following the methods described in Ref. [3]. Structures were investigated with the Pt atom density of $\operatorname{Pt}(110)-(1 \times 1)$ and at most one first layer row shifted half a lattice spacing along the $\mathbf{A}_{1}$ direction, as found in the experiment, and with adjustable displacements along $\mathbf{A}_{2}$ and $\mathbf{A}_{3}$. Only one structure was identified that involved a high-lying Pt row, consistent with the experiment (Fig. 1), and its energy per surface unit cell was within $0.27 \mathrm{eV}$ from the most stable structure found. Since this energy difference is comparable to the accuracy of density functional theory [21], the density functional theory results are supporting the possibility of such a highlying Pt row. An essential feature of the proposed structure is the presence of one carbonate ion $\left(\mathrm{CO}_{3}^{2-}\right)$ per unit cell, which stabilizes the high Pt row and thus the commensurate oxide monolayer.

In this work we have explicitly demonstrated the role of spontaneously formed surface oxides in a catalytic process under (semi) relevant pressure and temperature conditions. The surface oxides found here on $\mathrm{Pt}(110)$ have a significantly higher reaction rate than the original metallic surface. Our observations show that in situ measurements under actual reaction conditions are an absolute necessity for a meaningful investigation of the surface structure and chemical behavior of this model catalyst. Clearly, the presence and role of the commensurate oxide layer could not have been found either in experiments under UHV or in so-called pre- and post-reaction experiments.

The authors would like to thank E. Paiser, H. Isern, and L. Petit for their support at ID03. We are grateful to F. Besenbacher (Aarhus) for communicating the results of Ref. [3] prior to publication. This work is part of the research program of the Foundation for Fundamental Research on Matter (FOM) and was made possible by financial support from both NWO and ESRF. This work was further supported by the Dutch Technology Foundation (STW) and by the European Union, under Contract No. NMP3-CT-2003-505670 (NANO2). We also acknowledge the Danish Research Councils and the Dansk Center for Scientific Computing for providing the computational resources.

[1] P. B. Rasmussen et al., Rev. Sci. Instrum. 72, 3537 (2001).

[2] B. L. M. Hendriksen and J.W.M. Frenken, Phys. Rev. Lett. 89, 046101 (2002).

[3] W. X. Li et al., Phys. Rev. Lett. 93, 146104 (2004).

[4] C. Quiros et al., Surf. Sci. 522, 161 (2003).

[5] A. Stierle et al., J. Chem. Phys. 122, 044706 (2005).

[6] E. Lundgren et al., Phys. Rev. Lett. 92, 046101 (2004).

[7] M. D. Ackermann et al., Surf. Sci. 557, 21 (2004).

[8] S. Helveg et al., Nature (London) 427, 426 (2004).

[9] H. Bluhm et al., Top. Catal. 23, 99 (2003).

[10] B. L. M. Hendriksen et al., Surf. Sci. 552, 229 (2004).

[11] H. Over et al., Science 287, 1474 (2000).

[12] K. Reuter, M. Scheffler, Phys. Rev. Lett. 90, 046103 (2003).

[13] P. Bernard et al., Rev. Sci. Instrum. 70, 1478 (1999).

[14] E. Vlieg et al., Surf. Sci. 233, 248 (1990).

[15] T. Gritsch et al., Phys. Rev. Lett. 63, 1086 (1989).

[16] J. R. McBride et al., J. Appl. Phys. 69, 1596 (1991).

[17] All structure factors presented here are the square root of measured intensities, after background subtraction and correction for slit settings and other geometrical factors.

[18] S. Helveg et al., Surf. Sci. 430, L533 (1999).

[19] X. Su et al., J. Am. Chem. Soc. 119, 3994 (1997).

[20] J.A. Rodriguez and D. W. Goodman, Surf. Sci. Rep. 14, 1 (1991).

[21] P. J. Feibelman et al., J. Phys. Chem. B 105, 4018 (2001). 